\title{
Link Utilization Based Multicast Congestion Control
}

\author{
Manisha Manjul*, Rajesh Mishra, Joytsna \\ Department of Computer Science \& Engineering, School of I.C.T., Gauatam Buddha University, Greater Noida, India \\ Email: "manisham@gbu.ac.in, rmishra@gbu.ac.in, jyotsna.pro@gmail.com
}

Received June 2013

\begin{abstract}
Networking is the important part of the computer networks. Multicasting is also one of the essential areas of networking. In multicasting, sender can send information or data to the group of end receivers in a single transmission. It is a big issue because of more data demands of receivers which cause the congestion. Multicast is one of the crucial method to avoid congestion and make network perform stably. In this paper, we propose, link utilization algorithm to deal with multicast congestion control. We propose the equation to improve the utilization of the channel or link. The simulation results show that proposed work provide the better throughput of the network with respect to sending rate, queue size, packet loss ratio and time.
\end{abstract}

Keywords: Multicast; Congestion; Link Utilization; Congestion Control

\section{Introduction}

In data networking, network congestion occurs when packet arrival rate exceeds the outgoing link capacity, slow processing, bursty traffic, or due to insufficient memory to store arriving packets. The causes include for this is queuing delay, packet loss or blocking of new connections. Modern networks use the congestion avoidance techniques, measures, algorithms or protocols to try to avoid congestion collapse. Another method to avoid the negative effects of network congestion is implementing priority schemes, so that some packets are transmitted with higher priority than others. The priority method does not solve the congestion in network by themselves rather they help to alleviate the effects of congestion for some services. The other method for congestion control is of congestion degree concept. In this method we are defining some threshold value for the congestion occurrence and then calculate the Congestion degree. If the calculated congestion degree smaller than the predefined threshold the transmission rate is increased else decreased. Many ways had been given to control the congestion. Some basics are slow start, congestion avoidance, fast retransmission and fast recovery. The focusing section in the multicast congestion control, their approaches and protocols of these approaches of multicast congestion control: single rate and multi-rate [1]. Various mechanisms have been proposed to control congestion in network. Some mechanisms like TFMCC, LIMD, AIMD, PGMCC, proactive approach in which congestion degree concept

${ }^{*}$ Corresponding author. is used which helps in adjusting the sending rate of the source like that we also propose the congestion control algorithm based on link utilization. For this, propose the equations based on utilization to improve the channel usage. This algorithm improves the initial sending rate by giving the new sending rate for proper utilization of the network or link. The simulation of this algorithm is done using NS-2 tool and we have analyzed that throughput of the network with respect to the sending rate, queue size, packet loss ratio and time. We have observed that the proposed work provide the better throughput of the network.

\section{Related Work}

There are different mechanisms have been adopted till now to control the congestion in the network. We know that TFMCC [2] is a steady state equation based multicast technique to calculate the throughput of the network

$$
\begin{aligned}
& \mathrm{X}_{\mathrm{TCP}} \\
& =\mathrm{S} / \operatorname{RTT}\left((\operatorname{Sqrt}(2 \mathrm{p} / 3))+12 *(\operatorname{Sqrt}(3 \mathrm{p} / 8)) \mathrm{p}\left(1+32 \mathrm{p}^{2}\right)\right)
\end{aligned}
$$

Where $\mathrm{S}$ is Packet Size, $\mathrm{p}$ is Packet loss Ratio, $\mathrm{X}_{\mathrm{TCP}}$ is Throughput, and RTT is the Round trip time [3].

But it has some problems. First, it is slow in identifying the Congestion representative and therefore it is slow in reacting to changes in the congestion condition. Secondly, the CLR drag down the whole TFMCC session. Therefore, some modifications are made to TFMCC using Additive Increase Multiplicative Decrease (AIMD) 
approach by proposing a new equation to calculate the throughput [4-6]

$$
\mathrm{X}_{\mathrm{AIMD}}=\alpha / \mathrm{p}\left(\beta^{1 / 1-\mathrm{p}} / 1-\beta^{1 / 1-\mathrm{p}}\right)
$$

Where $\alpha=\mathrm{S} / \mathrm{RTT}, \beta$ is decreasing factor $(0<\beta<1)$

Now a proactive approach is used for congestion prevention. In this, the congestion is calculated as follows:

$$
\mathrm{F}_{\text {con }}=1-\chi_{\mathrm{n}} / \mathrm{d}
$$

Where $x_{n}=\mathrm{RTT}_{\mathrm{n}}-\mathrm{RTT}_{\mathrm{n}-1}$ and $\mathrm{d} \epsilon$ [RTT $\left.\mathrm{RTin}_{\min }, \mathrm{RTT}_{\max }\right]$. $\mathrm{RTT}_{\text {min }}$ and ${ }_{\mathrm{RTTmax}}$ correspond to the min. and max. RTT experienced within the multicast session.

Then, source adjusts its rate as

$$
\mathrm{X}_{\mathrm{n}+1}=\mathrm{X}_{\mathrm{n}} * \mathrm{f}_{\text {con }}
$$

Where $X_{n}$ is throughput before congestion, $X_{n+1}$ is throughput after congestion, $\mathrm{f}_{\mathrm{con}}$ is congestion factor. If, $X_{n}>0$ then source rate is decreased else increased [4].

Other way is that, if we want to improve the throughput of the network we use Logarithmic Increase multiplicative Decrease (LIMD) technique instead of AIMD technique for throughput calculations and show the better results. The LIMD equation as follows:

$$
\mathrm{X}_{\mathrm{LIMD}}=2^{\beta} \alpha\left(1+\beta \log _{2}\right)-\mathrm{p}
$$

Where $\alpha=\mathrm{S} / \mathrm{RTT}$, L is Packet Size, RTT is round Trip Time, $\mathrm{p}$ is Packet Loss Ratio, $\beta$ is Reduction factor.

Another way to control congestion in Wireless Sensor Network is Fuzzy approach by calculating the congestion degree $\left(C_{d}\right)$

$$
\left(\mathrm{C}_{\mathrm{d}}\right)=\mathrm{T}_{\mathrm{s}} / \mathrm{T}_{\mathrm{a}}
$$

Where $\mathrm{T}_{\mathrm{s}}=$ local packet inter-service time and $\mathrm{Ta}=$ local packet inter-arrival time.

Once congestion is detected, it is notified by using Implicit Congestion Notification (ICN) signaling. After receiving this signal the intermediate nodes adjust its sending rate so that congestion doesn't occur. Further congestion is implemented using Fuzzy logic controller [7,8].

Here we define some threshold value. If the congestion degree is greater than the defined threshold value, then congestion occurs and ICN signal is sent to intermediate nodes to inform that they adjust their sending rates; else there is no congestion in the network.

The other mechanism uses the Fuzzy-logic-based Rate Adaption (FRA) Scheme for TFMCC to control the congestion in order to enhance the smoothness of TFMCC. In order to alleviate oscillations of sending rate for TFMCC sender, FRA introduces five actions for adjusting rate [9-11].

These five actions are as follows:

a) MD (Multiplicative Decrease)

b) $\mathrm{AD}$ (Additive Decrease)

c) MI (Multiplicative Increase) d) AI (Additive Increase)

e) KP (Keep)

And uses the Fuzzy Controller to decide which action should be taken according to the feedback information from CLR (Current Limiting Receiver). In dynamic network environment, fuzzy controller uses the difference between expected rate and sending rate to reflect the congestion degree, as well as the difference between two latest consecutive expected rates to predict the trend of network. Under the fuzzy controller, KP, AD and MD actions eliminate the "sawtooth" phenomenon in TFMCC, which is crucial for smoothing sending rate [9]. When the available bandwidth is turning abundant, MI action can increase sending rate rapidly, making FRA have shorter responding time and can fully utilize the resource. In order to be friendly to TCP flows, the fuzzy controller has unsymmetrical membership functions and biased inference rules.

\section{Proposed Work}

We have seen that many algorithms have been proposed to control the congestion in the multicast network. These algorithms used different protocols to reduce congestion by adjusting the sending rate of the sender and different mechanisms has been proposed to indicate the congestion representative. The heterogeneous behavior of the network leads to the more utilization of bandwidth which results in congestion in the network. We propose an algorithm to improve the utilization by keeping the same sending rate while congestion occurs in the network. For this we have to first calculate the link utilization using existing link utilization method, then we again calculate utilization by our own proposed formula.

\section{Proposed Algorithm}

We are proposing an algorithm to improve the utilization by keeping the same sending rate while congestion occurs in the network. For this we have to first calculate the link utilization using old link utilization method. The proposed algorithm entitles LUMCC is given below:

Algorithm: Link Utilization Based Multicast Congestion Control (LUMCC)

1) Initialize the total link capacity.

2) Initialize the initial sending rate.

3) Initialize the queue size.

4) Initialize the packet size.

5) Set the session time.

6) Calculate the packet loss ratio on the link.

$$
P_{l s}=P_{d} / P_{d}+P_{s}
$$

Where $P_{l s}$ is the Packet loss observed on the link, $P_{d}$ is the number of Packets dropped, $P_{s}$ is the number of Packets sent on the link. 
7) Calculate the link utilization, $\alpha_{i j}$.

$$
\alpha_{i j}=\Sigma b w_{f^{*}} \max \left(X_{i f}\right)^{f} / C_{i j} \text { for all } f C F
$$

Where $\Sigma b w_{f^{*}}\left(X_{i f}\right)^{f}$ denotes the value of total traffic demand for all flows $f E F$ that are transmitted through link $(i, j), C_{i j}$ is the link capacity.

8) Setting the threshold values:

a) If $\left(99 \%\right.$ of link utilization $\left.<\alpha_{i j}\right)$

Then, Congestion is very high and we adjust

$$
F_{\text {new }}=\alpha_{i j} / \beta * 2
$$

b) If $\left(99 \% \leq \alpha_{i j}<90 \%\right)$

Then, Congestion is high and we adjust

$$
F_{\text {new }}=\alpha_{i j} * 2^{\beta} / 2
$$

If (Fnew > 99\%)

Then go to step " $a$ ".

c) If $\left(90 \% \leq \alpha_{i j} \leq 50 \%\right)$

Then, Congestion is medium and we adjust

$$
F_{\text {new }}=\alpha_{i j} * \beta * 2
$$

If (Fnew $>90 \%)$

Then go to step " $b$ ".

d) If $\left(0<\alpha_{i j}<49 \%\right)$

Then, Congestion is low and we adjust

$$
F_{\text {new }}=\alpha_{i j} * \log _{2} \alpha
$$

Else increment $F_{\text {new }}$ till its value reaches to medium value.

We see the example of propose algorithm given as below:

Example: Suppose the Link Capacity is $100 \mathrm{Mbps}$, Initial Sending Rate is $80 \mathrm{Mbps}$, Packet size is 300, RTT is $150 \mathrm{~ms}$, decreasing factor, $\beta$ is $0.65(0<\beta<1)$, increasing factor $\alpha=\mathrm{S} / \mathrm{RTT}$ is 2 and we vary the Queue size.

Case 1: Queue size $=50$ packets

Link utilization,

$$
\begin{aligned}
\alpha_{\mathrm{ij}} & =\Sigma \mathrm{bw}_{\mathrm{f} *} \max \left(\mathrm{X}_{\mathrm{if}}\right)^{\mathrm{f}} / \mathrm{C}_{\mathrm{ij}} \mathrm{f} \epsilon \mathrm{F} \\
& =80 * 50 / 100=40 \% .
\end{aligned}
$$

The link utilization is $40 \%$ means that congestion is low. Then we use third condition and the proposed formula is:

$$
\begin{aligned}
F_{\text {new }} & =\alpha_{i j} * \log _{2} \alpha \\
& =40 * \log _{2}(\mathrm{~S} / \mathrm{RTT})=40 * \log _{2}(2)=40 \%
\end{aligned}
$$

Again, the $\mathrm{F}_{\text {new }}$ is $40 \%$, then we go to step "c".

$$
F_{\text {new }}=\alpha_{i j} * \beta * 2=40 * 0.65 * 2=52=65 \%
$$

So, our utilization comes to $65 \%$.

Case 2: Queue size $=80$ packets

Link utilization,

$$
\begin{aligned}
\alpha_{\mathrm{ij}} & =\Sigma \mathrm{bw}_{\mathrm{f}} * \max \left(\mathrm{X}_{\mathrm{if}}\right)^{\mathrm{f}} / \mathrm{C}_{\mathrm{ij}} \text { for all f } \epsilon \mathrm{F} \\
& =80 * 80 / 100=64 \% .
\end{aligned}
$$

The link utilization is $64 \%$ means that congestion is medium. Then we use second condition and the proposed formula is:

$$
F_{\text {new }}=\alpha_{i j} * \beta * 2=64 * 0.65 * 2=83 \%
$$

So, our utilization comes to $83 \%$.

Case 3: Queue size $=120$ packets

Link utilization,

$$
\begin{aligned}
\alpha_{\mathrm{ij}} & =\Sigma \mathrm{bw}_{\mathrm{f} * \max }\left(\mathrm{X}_{\mathrm{if}}\right)^{\mathrm{f}} / \mathrm{C}_{\mathrm{ij}} \text { for all } \mathrm{f} \epsilon \mathrm{F} \\
& =80 * 120 / 100=96 \% .
\end{aligned}
$$

As utilization is $96 \%$ which shows the high congestion in the network is according to set thresholds. So, we have made the congestion medium. For we use first condition and the proposed formula is:

$$
F_{\text {new }}=\alpha_{i j} * 2^{\beta} / 2=96 * 2^{0.65} * 2=5 \%
$$

We conclude that if our link utilization is high then we need more care about the congestion, otherwise regularly needs to increase the flow speed according to low and medium include with medium and high factor of speed respectively. Therefore, utilization of link is very important phenomenon for control the congestion.

\section{Result and Discussion}

\subsection{Simulation Topology}

The simulation is done on NS-2 tool. We created a network topology scenario to simulate our work. Our aim is to make a simulation environment for single source multicasting. The parameters used for the multicast topology are given below (Table 1).

For the multicast topology, Node 0 is a single source and box 1 is a router. Where node 2, 3, 4, 5 and 6 are the group receivers through which nodes 7, 8, 9 are attached to group 2, nodes $10,11,12$ are attached to group 3,13 ,

Table 1. Simulation Parameters.

\begin{tabular}{cc}
\hline Parameters & Value \\
\hline Link Bandwidth & $10-100 \mathrm{Mbps}$ \\
Link Delay & $20 \mathrm{~ms}$ \\
Queue Size & $50-99$ packets \\
Sending Rate (initial) & $80 \mathrm{Mbps}$ \\
No. of Groups & 5 \\
No. of Receivers & 15 \\
RTT & $150 \mathrm{~ms}$ \\
Packet Size & 300 \\
Session Time & $500 \mathrm{~ms}$ \\
$\mathrm{Th}_{\text {LU }}$ (Congestion Status) & $59 \%$ above (very High), $99 \%-90 \%$ (High), \\
\hline
\end{tabular}


14, 15 are attached to group 4, nodes $16,17,18$ are attached to group 5 and nodes 19, 20, 21 are attached to group 6 as an individual receiver respectively. The network topology for single multicast is below of 15 receivers:

The sender and router supports $1.5 \mathrm{Mb}$, node (n1-n2, n1-n3, n1-n4, n1-n5 and n1-n6) also supports 1.5 Mb with delay of $10 \mathrm{~ms}$ and nodes (n2-n7, n2-n8, n2-n9, n3-n10, n3-n11, n3-n12, n4-n13, n4-n14, n4-n15, n5-n16, n5-n17, n5-n18, n6-n19, n6-n20 and n6-n21) supports 1.0 $\mathrm{Mb}$ with a delay of $5 \mathrm{~ms}$.

\subsection{Result and Comparison}

Figure 2 shows the comparison of throughput for different values of Queue Size at $\beta=0.65$. The variation is due to the link utilization of the network. The new proposed sending rate $\left(F_{\text {new }}\right)$ shows the better result than the existing approach $\left(a_{i j}\right)$ because of the less packet loss ratio. At 150 Queue size the throughput of existing approach is more than the proposed approach because according to our condition more than $90 \%$ link utilization shows the high congestion. So, we reduce this congestion to medium i.e. less than $90 \%$. That's why here the result of proposed approach is less than existing approach.

Figure 3 shows the variation of packet loss ratio with the throughput. As the graph shows that the throughput and packet loss are proportional to each other. Because

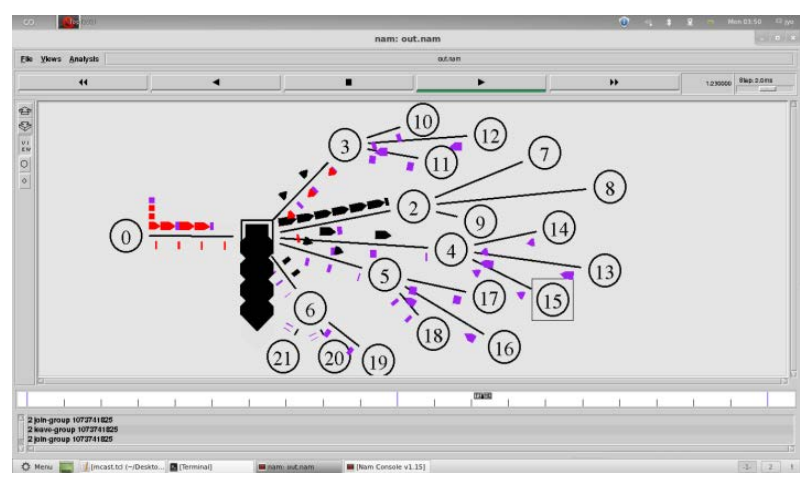

Figure 1. Multicast Topology.

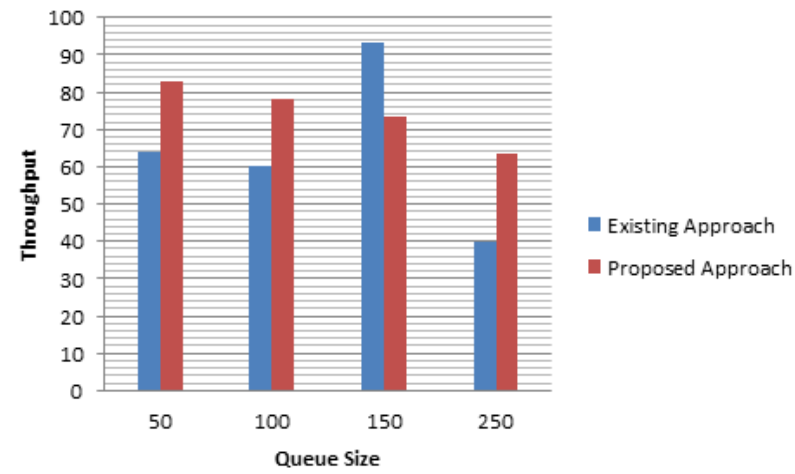

Figure 2. Throughput Vs Queue size. there is a less packet loss than the existing approach due to congestion control based on link utilization while other is based on normal equation which is based on packet size and packet loss.

Figure 4 shows the variation of Initial sending rate with the throughput. The graph shows that sending rate and throughput is directly proportional to each other. The throughput increases with the increment in the $\beta$ value.

Figure 5 Shows the variation of throughput with the time. It shows the maximum time needed for the multicast source till reaching a steady state throughput. It is clear that proposed approach Outperforms existing approach.

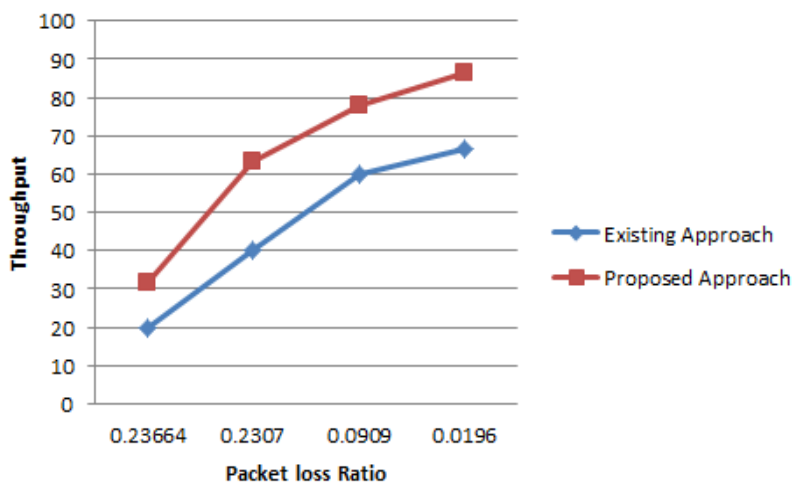

Figure 3. Variation of Packet Loss Ratio with the Throughput.

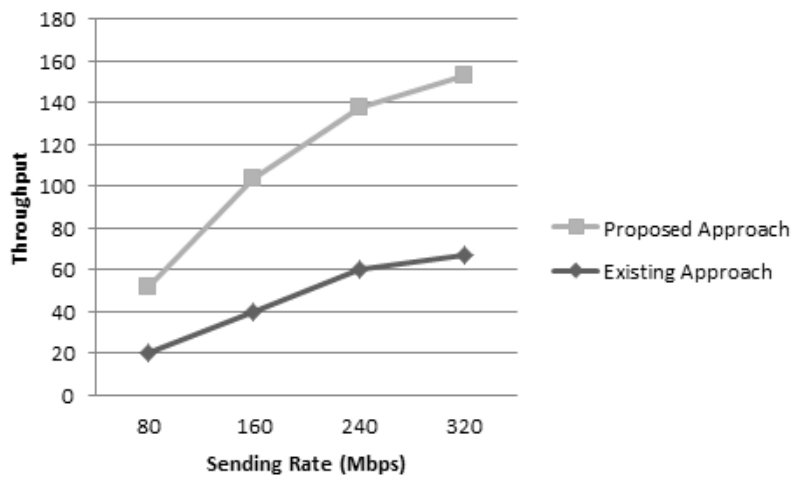

Figure 4. Variation of Sending Rate with Throughput.

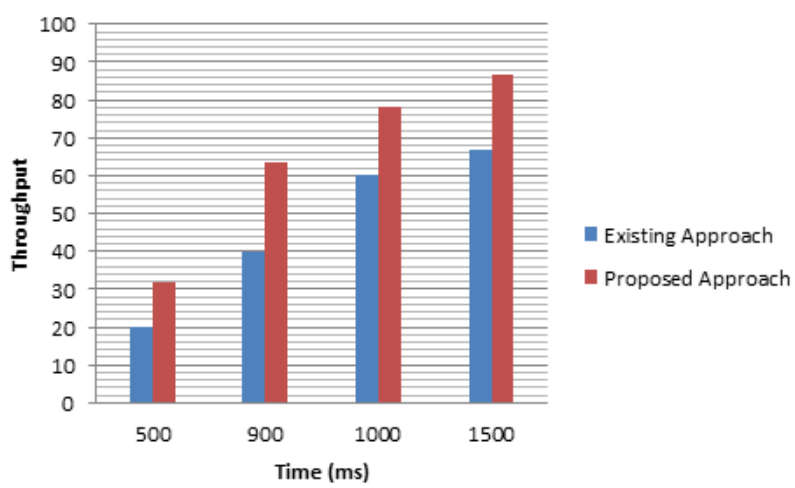

Figure 5. Variation of Throughput with Time. 


\section{Conclusion}

In this paper, we propose a multicast congestion control mechanism to improve the utilization of the network. The main part of our research is an algorithm for congestion control which based on utilization of link and taking decision according to high, medium and low congestion. The proposed equations will be defined after setting the threshold value. The equations are based on utilization to improve the channel usage. This algorithm improves the initial sending rate by giving the new sending rate for proper utilization of network. The simulation of this work is done using the Network Simulator tool (NS-2) and we have analyzed the throughput of the Network with respect to sending rate, queue size, packet loss ratio and time. We have observed that proposed work provide the better throughput of the network.

\section{REFERENCES}

[1] S. Kumar and K. Singh, "Efficient Single Rate Based Multicast Congestion Control," IJCA Proceedings on National Conference on Innovative Paradigms in Engineering and Technology, 2012.

[2] S. Shi and M. Waldvogel, "A Rate-Based End-to-End Multicast Congestion Control Protocol," International Conference on Convergence and Hybrid Information Technology, 2000.

[3] W. Kammoun and H. Youssef, "Improving End to End Multicast Rate Control in Networks," IEEE Proceedings of Computer Communication ISCC, 2009.

[4] W. Kammoun and H. Youssef, "Improving the Performance of End-to-End Single Rate Multicast Congestion
Control," Proceedings of Computer and Communication, 6-9 July 2008.

[5] G.-I. Kwon and J. W. Byers, "Leveraging Single Rate Schemes in Multiple Rate Multicast Congestion Control Design," IEEE Journal on Selected Areas in Communications, Vol. 22, No. 10, 2004, pp. 1975-1986. http://dx.doi.org/10.1109/JSAC.2004.836006

[6] M. G. abowski, M. Stasiak and P. Zwierzykowski, "Multicast Connections in a Virtual Circuit Switching Node," Proceedings of the Advanced Industrial Conference on Telecommunications/Service Assurance with Partial and Intermittent Resources Conference/ELearning on Telecommunications Workshop, 2005.

[7] R. Chakravarthi and C. Gomathy, “A Fuzzy Approach To Detect And Control Congestion In Wireless Sensor Networks," Indian Journal of Computer Science and Engineering (IJCSE), Vol. 3, No. 3, 2011, pp. 476-483.

[8] J. Bian, Z.-Q. Wang, H.-H. Ke and G.-Z. Zhang, “Congestion Control Protocol Based on Delay Parameters for Layered Multicast Communication,” ICCT International Conference on Communication Technology, 10 April 2007.

[9] H. Ma, X. Meng, L. Zhou, H. Li and X. Zhang, "FuzzyLogic-Based Adaption Scheme for TFMCC,” Proceedings of International Conference on Networking and Digital Society, 2009.

[10] Y.-W. Leung, "Congestion Control for Multipoint Video Conferencing," IEEE Transactions on Circuits and Systems for Video Technology, Vol. 10, No. 5, 2000.

[11] X. Zhang, C. R. Li, X. Li and C. X. Bao, "Multicast Congestion Control on Many-to-Many Videoconferencing," Second International Conference on Future Generation Communication and Networking, 30 December 2008. 